\title{
Lidil
}

Revue de linguistique et de didactique des langues

$61 \mid 2020$

Le mépris en discours

\section{Une forme insidieuse de mépris : les micro- agressions linguistiques en Nouvelle-Calédonie}

An Insidious Form of Contempt: Linguistic Micro-Aggressions in New Caledonia

Elatiana Razafi et Fabrice Wacalie

\section{(2) OpenEdition}

1 Journals

Édition électronique

URL : http://journals.openedition.org/lidil/7477

DOI : 10.4000/lidil.7477

ISSN : 1960-6052

Éditeur

UGA Éditions/Université Grenoble Alpes

Édition imprimée

ISBN : 978-2-37747-195-9

ISSN : $1146-6480$

Référence électronique

Elatiana Razafi et Fabrice Wacalie, « Une forme insidieuse de mépris : les micro-agressions linguistiques en Nouvelle-Calédonie », Lidil [En ligne], 61 | 2020, mis en ligne le 02 mai 2020, consulté le 02 mai 2020. URL : http://journals.openedition.org/lidil/7477 ; DOI : https://doi.org/10.4000/lidil. 7477

Ce document a été généré automatiquement le 2 mai 2020.

(c) Lidil 


\title{
Une forme insidieuse de mépris : les micro-agressions linguistiques en Nouvelle-Calédonie
}

\author{
An Insidious Form of Contempt: Linguistic Micro-Aggressions in New Caledonia
}

Elatiana Razafi et Fabrice Wacalie

\section{Introduction}

1 La présente contribution propose l'interprétation d'un matériau biographique et photographique élaboré lors d'un projet pédagogique et scientifique intitulé «AK-100 : ces accents qui dérangent ». Il a été mené en 2018 avec des étudiants de l'Université de la Nouvelle-Calédonie (désormais UNC), soit dans un archipel du Pacifique Sud qui donne à voir un "paradis pour la diversité linguistique " (Razafimandimbimanana, 2017). Il est vrai qu'on y dénombre une centaine de langues, majoritairement issues de familles océaniennes, austronésiennes, austroasiatiques et européennes. Pourtant, l'immersion dans le quotidien urbain ne donne ni à voir ni à entendre le foisonnement linguistique ${ }^{1}$ de cette diversité. La langue française domine à l'université comme en ville (espaces médiatiques, lieux de consommation, paysage sociolinguistique). De ce point de vue, la diversité linguistique semble être réservée, voire confinée, au domaine du privé ou encore aux espaces interactionnels informels. Dans tous les cas, elle fait l'objet d'un débat continu quant à la place qu'elle devrait occuper dans le paysage langagier, dans les espaces publics ou encore à l'école.

Le statut de collectivité sui generis française ${ }^{2}$ permet d'expliquer cette distribution inégale - le français est la seule langue dotée du statut de «langue officielle »-, mais cela ne peut suffire pour rendre compte des valeurs ambigües assignées aux différences inter et intralinguistiques au sein de ce territoire.

En raison des hiérarchisations qui se jouent entre les différentes langues et variétés linguistiques en présence, il en résulte en effet une relation de «culte» et de 
«mépris ». En accord avec Honneth (2006), nous situons le mépris au cœur de l'expérience sociale en ce qu'il conditionne les sentiments de reconnaissance et, de ce fait, d'appartenance. Nous nous intéresserons au mépris observé sous forme de «micro-agressions linguistiques» (Razafimandimbimanana \& Wacalie, 2019; Frain, Razafimandimbimanana \& Wacalie, 2019), soit des remarques insidieuses qui, du point de vue des destinataires, sont vécues comme la subordination d'expériences sociales en raison de pratiques langagières dévalorisées.

4 Après une interprétation du terrain calédonien en matière de rapports sociaux aux langues, nous rendrons compte des micro-agressions linguistiques du point de vue de leur réception. En l'occurrence, il s'agit des témoignages de 57 étudiants de l'UNC (30 inscrits en Licence 2, filière Lettres ou Langues et Cultures océaniennes; 27 étudiants en Licence 3, parcours Didactique du plurilinguisme). Une approche qualitative du matériau empirique (autoportraits et récits) permettra d'illustrer les micro-agressions linguistiques que ces étudiants assimilent à des effets méprisants. Nous verrons enfin qu'une certaine distanciation est rendue possible à l'aide de médiations artistiques.

\section{La Nouvelle-Calédonie, héritière et productrice d'inégalités linguistiques}

5 Repensées en tant qu'espaces sociaux, les langues sont le lieu des (re)négociations des conditions d'appartenance et d'accès aux ressources sociétales. Or, les rapports de pouvoir ne sont ni donnés ni sans effets. Pour nous, l'analyse des pratiques langagières est donc concomitante avec la prise en compte des effets socio-symboliques tels qu'exprimés par les acteurs en question et du contexte sociohistorique dans lequel ils évoluent.

\subsection{Un territoire en phase de réappropriation linguistique}

6 Parmi une quarantaine ${ }^{3}$ de langues kanak dénombrées, seules quatre figurent dans les programmes scolaires du second degré en tant que langues optionnelles au baccalauréat, et ce depuis $1992^{4}$. Se voient ainsi entretenues les inégalités linguistiques causées par le traitement colonial de la diversité linguistique - l'école était alors le siège d'un impératif français agissant, en premier lieu, au détriment des langues kanak (Salaün, 2005; Fillol \& Vernaudon, 2011; Colombel \& Fillol, 2012; Razafimandimbimanana \& Favard, 2018; Razafimandimbimanana, à paraitre). Aujourd'hui ${ }^{5}$, nos observations du terrain nous amènent à penser que la NouvelleCalédonie est dans une phase de réappropriation linguistique. L'année 2019 correspond d'ailleurs au $20^{\mathrm{e}}$ anniversaire de la filière «Langues et Cultures océaniennes » offerte à l'Université de la Nouvelle-Calédonie, une orientation que les étudiants expliquent non seulement par l'envie de mieux se connaitre mais également par une volonté décentrée de contribuer à la transmission des langues et cultures du pays ${ }^{6}$. Le foisonnement du milieu artistique est un autre indicateur de cette dynamique collective entreprise pour avancer vers une meilleure acceptation de l'interculturalité du territoire. Si des actions de réappropriation sont à l'œuvre, la question des inégalités linguistiques reste vive, d'autant que se multiplient les normes de référence et, conséquemment, les sources de tensions. 


\subsection{Polarisation des normes linguistiques versus sédentarisation de mythes nativistes}

7 Deux mouvements opposés s'observent généralement lorsqu'il s'agit des rapports sociaux aux langues. D'une part, la polarisation des normes et des références regroupées autour de Nouméa ${ }^{7}$, centre urbain localisé en Province Sud où se concentre plus de $74 \%$ de la population. Comparativement, la Province Nord comprend $19 \%$ de la population tandis que les iles Loyauté en comptent seulement 7 \% (INSEE, 2015). D'autre part, la sédentarisation de mythes nativistes avec le culte de certaines pratiques hors Nouméa perçues plus «vraies ", " pures » ou " authentiques ». Comme nous le verrons plus loin, les étudiants disent parfois connaitre un « locuteur natif » correspondant à la définition du locuteur-auditeur idéal de Noam Chomsky :

Appartenant à une communauté linguistique complètement homogène, qui connait parfaitement sa langue et qui, lorsqu'il applique en une performance effective sa connaissance de la langue n'est pas affecté par des conditions grammaticalement non pertinentes, telles que limitation de mémoire, distractions, déplacement d'intérêt ou d'attention, erreurs (fortuites ou caractéristiques). (Chomsky, 1971, p. 12)

8 Nous parlons de mythe en nous fondant sur des sources scientifiques et empiriques. Le terme locuteur natif a effectivement donné lieu à un "demi-siècle de critiques " entre autres dans la littérature anglophone (Muni Toke, 2013, p. 5) :

Le terme «natif» est donc également porteur d'une valeur ethnico-raciale implicite : l'authenticité et la légitimité du locuteur natif tiennent à l'appartenance culturelle qu'on lui prête - voire à son phénotype [...]. On voit quels raccourcis il a soudainement fallu accepter, pour que la « compétence » supposée d'un locuteur se retrouve corrélée à son origine ethnique. Et pourtant, la construction d'inégalités sociales à partir de ce présupposé est monnaie courante, et il y aurait bien ainsi des natifs qui seraient plus natifs que d'autres. (Muni Toke, 2013, p. 6)

9 Ce mythe nativiste trouve aussi résonance sur le terrain calédonien. À en croire des habitants de l'ile d'Ouvéa, par exemple, le locuteur-auditeur idéal existe bel et bien. Toutefois, leurs descriptions indiquent le plus souvent des locuteurs proprement « introuvables » car soit décédés, soit situés dans des lieux davantage isolés sur l'ile (Dotte, 2019). Nous retrouvons cette idéalisation dans les représentations des étudiants. À la question «Pensez-vous connaitre une personne pouvant parfaitement correspondre au profil du locuteur-auditeur idéal tel que défini par Noam Chomsky?", nombreux étaient ceux en début de programme 8 à répondre par l'affirmative :

Mon père, âgé de 55 ans et originaire de Canala correspond parfaitement à la définition de "locuteur natif » tel que défini par Noam Chomsky. Il est locuteur du xârâcùù depuis son plus jeune âge. [...] Il est entouré d'une famille homogène, c'està-dire qu'il est issu d'une famille où le xârâcùù est à la source de toute communication. (Étudiante, Licence 2)

10 Sans surprise, les sentiments provoqués par la croyance en l'existence de tels locuteurs-auditeurs idéaux incluent l'insécurité linguistique, la non-légitimité en tant que membre de la communauté linguistique, le mutisme et, pour certains, le refus de transmission aux plus jeunes générations. La quasi-totalité des étudiants ne se considère pas comme des "locuteurs natifs" tant ce statut leur semble être inaccessible. Pour eux, il y a une polarisation des références avec une double injonction impossible à satisfaire : « devoir bien parler le français » et « devoir parler en langue " 9 . 
Leurs récits font aussi (ré)apparaitre des micro-évènements vécus depuis leur enfance et les premières étapes de scolarisation. Ils font part de jugements de valeur qui portent sur leur accent ou leur manière de parler. Le mépris dont ils disent faire l'objet est diffusé et entretenu à travers ce que nous appelons des «micro-agressions linguistiques".

\section{Les « micro-agressions linguistiques » : un concept spécifique au mépris insidieux}

11 Un des objectifs du projet AK-100 est d'œuvrer pour une meilleure reconnaissance de l'écosystème langues-cultures-identités, lui-même dépendant de la diversité linguistique et sociale. C'est ainsi que l'analyse des récits d'étudiants de la NouvelleCalédonie, majoritairement plurilingues, a donné lieu au concept de «micro-agression linguistique ». Il aide à mieux comprendre ce que des éléments discursifs plus ou moins implicites produisent d'inégalitaire.

\subsection{Un concept social pour un projet social}

12 Au-delà de leur fonction communicationnelle, nous admettons les langues en tant qu'espace social où se (re)négocient les rapports de force et où se (dé)construisent les marqueurs identitaires. Dans cette perspective, la variation linguistique devient alors productrice de tensions, de revendications et de phénomènes de rejet; elle est un instrument dont s'arment les acteurs sociaux pour signifier leurs sentiments d'identification tout autant que leurs projets de différenciation. Plusieurs concepts existent déjà dans la littérature scientifique pour désigner les discriminations sociales basées sur des pratiques linguistiques dont « linguicisme» (Skutnabb-Kangas, 1986; Phillipson, 1992), " glotto-stéréotypie » (Bochman, 2001) et " glottophobie » (Blanchet, 2016). À travers celui de "micro-agression linguistique", nous souhaitons mettre l'accent sur le caractère banal et banalisé d'idéologies puristes.

13 La première publication du terme micro-agression est attribuée au docteur Chester Pierce (Pierce, Carew, Pierce-Gonzalez \& Willis, 1978, p. 66) avec l'expression microagression raciale. Cette dernière désigne des échanges "subtils, acerbes, souvent inconscients ${ }^{10}$ " qui se révèlent être des formes verbales ou non verbales de dévalorisation. D'apparence simple, les micro-agressions raciales, lorsqu'elles ponctuent le quotidien des personnes appartenant à des minorités sociales, produisent des effets conséquents et durables. Par exemple, elles agissent «au détriment des personnes dites de couleur noire parce qu'elles invalident leurs potentialités dans une multitude de situations en minant l'énergie psychique et spirituelle des récepteurs et en créant des iniquités ${ }^{11} »$ (Sue, 2004 cité dans Sue, Capodilupo, Torino \& Bucceri, 2007, p. 273).

Inspirées de cette théorisation, les «micro-agressions linguistiques » nous permettent plus spécifiquement d'étudier les remarques aux apparences trompeuses pour au moins deux raisons. Premièrement, elles paraissent flatteuses en surface (ex. «tu parles bien français...») mais deviennent stigmatisantes à la réception/réflexion (" ... pour un Kanak »). Deuxièmement, étant le reflet des stéréotypes et idéologies dominants, leur banalisation leur octroie un pouvoir véritable agissant. Loin d'être anodines, les microagressions identifiées par les étudiants les affectent pernicieusement. Ils disent se 
sentir évalués à répétition en raison de traits linguistiques non reconnus car jugés " incorrects ", "bizarres ", " illégitimes » ou encore "impurs ». Nous retrouvons bien là des expériences sociales du mépris qui «s'accompagne(nt) toujours de sentiments susceptibles de révéler à l'individu que certaines formes de reconnaissance sociale lui sont refusées » (Honneth, 1992, p. 166). Si, du point de vue des énonciateurs, les microagressions linguistiques paraissent ordinaires voire bien intentionnées ${ }^{12}$, l'analyse de leurs réceptions montre que l'accumulation et l'intériorisation de ces formes discursives de mépris enclenchent un "processus lent mais opératoire où le mépris de l'altérité linguistique cause l'abandon des langues» (Razafimandimbimanana \& Wacalie, 2019).

La démarche interprétative que nous adoptons part de l'expérience et de la subjectivité des étudiants. C'est au titre de destinataires de micro-agressions linguistiques qu'ils sont les seuls à véritablement pouvoir éprouver les sentiments négatifs qu'elles créent et donc à leur donner une réalité spécifique. Cette précision est importante car les mots ne sont pas (in)offensifs en soi. Il laisse aussi entendre que si certaines remarques nous semblent peu problématiques alors qu'elles sont vécues de manière menaçante par les étudiants, cela s'explique :

Dans la mesure où l'expérience de la reconnaissance est une condition dont dépend l'identité personnelle dans son ensemble, l'absence de cette reconnaissance, autrement dit le mépris, s'accompagne nécessairement du sentiment d'être menacé dans sa propre personnalité. (Honneth, 2006, p. 193)

Les étudiants décrivent des micro-agressions aux effets non immédiatement visibles mais qui, à la longue, provoquent une " paralysie » sociale et interactionnelle :

Les gens me font penser que je suis en insécurité langagière, alors je recherche cette perfection, qui malheureusement modifie mon identité, me crée un faux profil. La présence de cet accent involontaire et dont je ne me rendais pas compte auparavant me paralyse, je réfléchis à comment je dois m'exprimer, je pense en français et je traduis en drehu alors qu'en tant que «locutrice», cela doit être naturel et fluide ${ }^{13}$ ! (Étudiante, Licence 2)

Nous pouvons dire que les micro-agressions linguistiques sont des formes insidieuses de mépris social qui se manifestent diversement sur le plan discursif.

\subsection{Un concept motivé par le contexte calédonien}

18 Nos interprétations croisent plus exactement quatre formes distinctes d'accès au terrain :

1. des photographies produites par les étudiants à la suite d'une consigne les invitant à tirer leur autoportrait en affichant, sur une pancarte tenue face à l'objectif, une micro-agression linguistique dont ils peuvent témoigner en tant que destinataires;

2. des textes explicatifs dans lesquels les étudiants mettent en récit les situations dans lesquelles ils étaient/sont la cible des micro-agressions linguistiques montrées ainsi que les effets de celles-ci ;

3. différents travaux réflexifs réalisés par les étudiants au cours de leur formation (biographies langagières, écrits réflexifs, carnets de bord, portfolio réflexif);

4. nos observations davantage informelles et quotidiennes en tant qu'enseignants.

19 L'analyse croisée de ces différents observables nous font dire que les productions des étudiants sont sans équivoque : il y a des besoins réels de (re)valorisation de la diversité linguistique en Nouvelle-Calédonie. Cela a motivé la création du projet «AK-100 : ces 
accents qui dérangent» ainsi que la conceptualisation des micro-agressions linguistiques. Il en ressort une soixantaine d'autoportraits photographiques ${ }^{14}$, tous réalisés en 2018 à l'occasion d'une activité réflexive autour du concept de «locuteur natif ». L'objectif était de mettre les idéalisations théoriques du locuteur-auditeur idéal (Chomsky, 1971) à l'épreuve des vécus des étudiants que nous accompagnons en cours de Psycholinguistique (Licence 2) et au sein du parcours Didactique du plurilinguisme (Licence 3). Notons aussi que les étudiants qui y ont participé sont au moins bilingues (français, anglais ; français, une langue kanak ; français, une langue océanienne).

Or, effet collatéral du mythe nativiste évoqué plus haut, aucun d'entre eux ne s'estimait digne d'être décrit comme un «locuteur natif» dans la mesure où ils ne pouvaient prétendre ni être originaires d'une "communauté linguistique homogène", ni connaitre "parfaitement » une langue, ni même être à l'abri de «fautes ». Interpellés par les perceptions dévalorisantes que les étudiants portaient sur leurs propres compétences langagières, nous avons voulu en apprendre davantage. Les premières discussions sur le sujet avec les étudiants ${ }^{15}$ ont permis de voir à quel point leurs récits convergeaient autour d'inhibitions en tant que sujets-locuteurs. Tantôt, le conflit intergénérationnel resurgit ( les vieux nous reprochent de ne pas respecter la langue ${ }^{16}$ $»$ ), tantôt, ce sont leurs pratiques plurilingues qui posent problème ( $Q$ Quand tu prononces des mots en langue, ça se voit que t'es une RATÉE! », photo ${ }^{\circ} 1$ ). Pour ceux qui s'attribuent un répertoire monolingue, ce sera justement ce qui déclenchera des formes d'ethnicisation et d'exclusion sociale: «Tu es blanche donc tu parles qu'une langue !» (photo $\mathrm{n}^{\circ} 2$ ). La finalité sociale était la création d'un espace artistique urbain à travers lequel les étudiants pouvaient renvoyer un contre-discours face au mépris linguistique ambiant, fil rouge des ressentis de leurs récits textuels et photographiques.

Photo $n^{\circ} 1$.

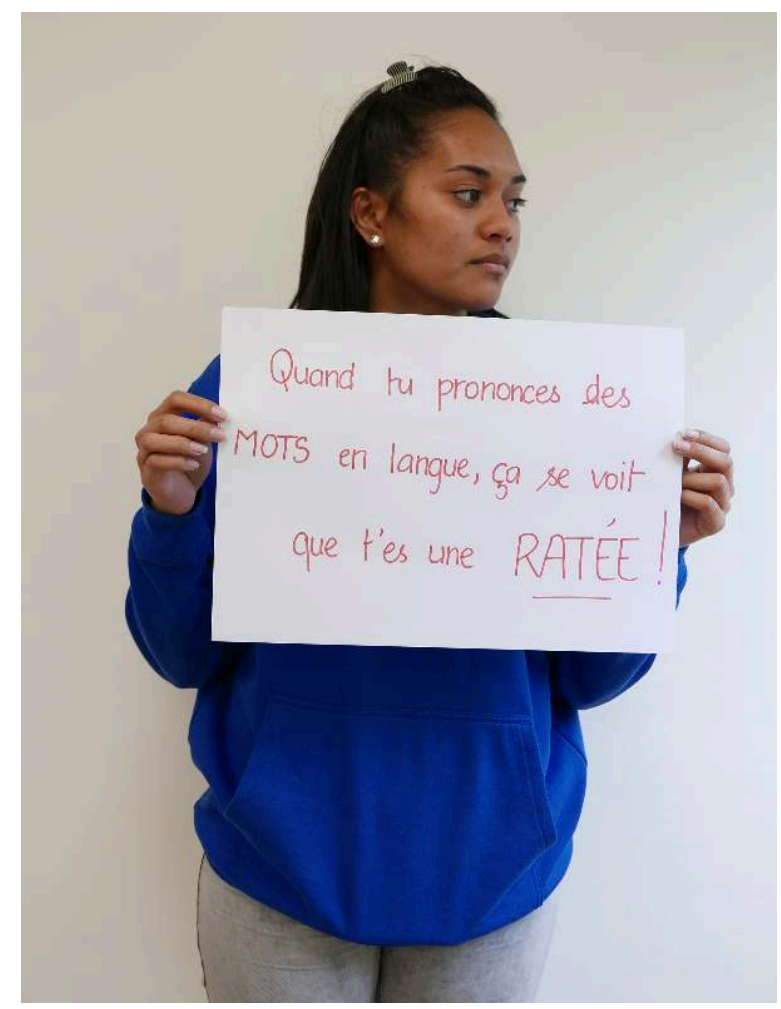


Photo $n^{\circ} 2$.

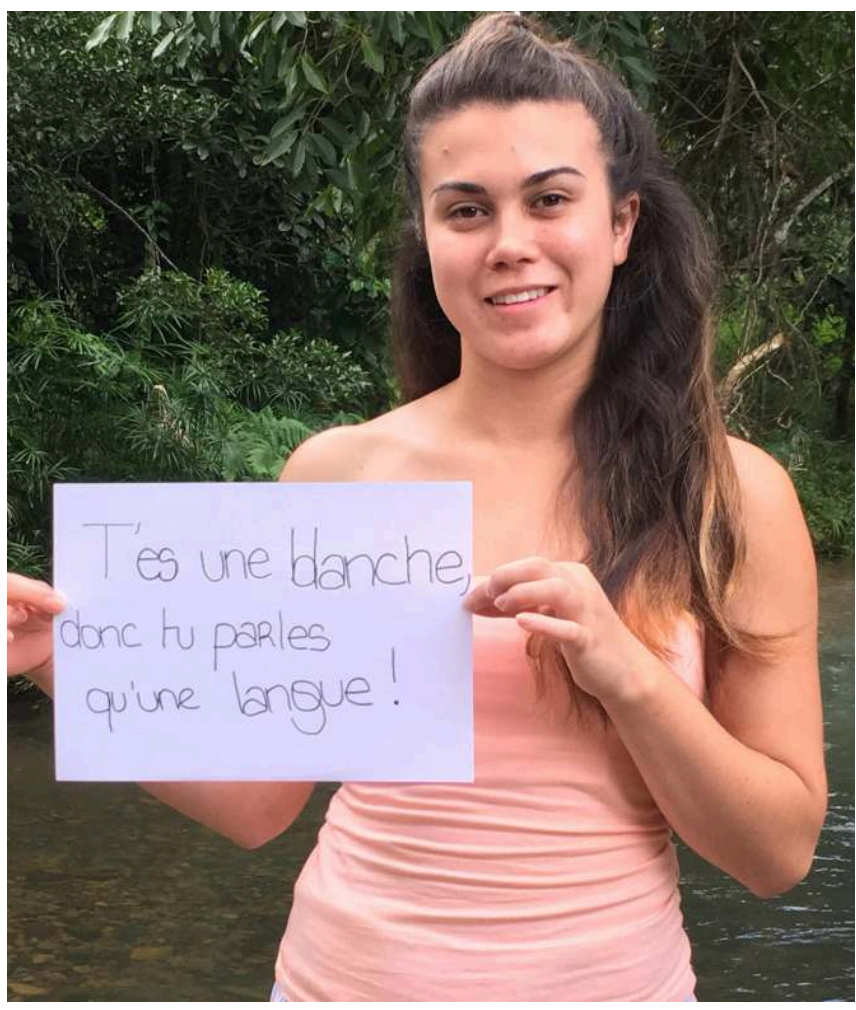

\subsection{Un concept pour identifier les formes de mépris linguistique}

21 Les micro-agressions linguistiques, parce que banalisées, participent à l'intériorisation d'images méprisantes reléguant l'altérité linguistique aux frontières d'un «nous » fantasmé comme étant homogène, authentique et en cela, de valeur supérieure aux autres. Selon nos analyses, les étudiants évoquent le mépris sous trois formes.

Premièrement, le mépris relève d'un processus de disqualification interlinguistique qui alimente le sentiment d'exclusion sociale en raison d'une hiérarchisation des systèmes linguistiques. Le plus souvent, nous retrouvons les représentations qui positionnent le français au sommet de la stratification sociale (Barnèche, 2005 ; Salaün, 2005 ; Colombel \& Fillol, 2012; Vernaudon, 2013). Hérité des politiques assimilationnistes menées depuis la fin du régime de l'indigénat (1946) jusqu'au début des années 1970, le mépris interlinguistique obéit à des mécanismes de reproduction conceptuelle et organisationnelle. Les schémas de dominations sociales du passé se voient réactivés avec, par exemple, la pensée coloniale et la non-reconnaissance des droits linguistiques des communautés kanak. L'attribution même du statut de « langue » demeure ambigüe avec une distinction (encore) opératoire entre «langue » et " dialecte». Entre autres, les entreprises de description linguistique reproduisent et diffusent cette distinction pourtant problématique, du moins quand la langue est admise comme une pratique sociale à part entière et que sont considérées prioritaires les effets susceptibles d'être engendrés sur les locuteurs. Preuve du poids institutionnel de cette distinction, l'Académie des langues kanak, établissement du gouvernement de la NouvelleCalédonie, présente sa mission première comme devant assurer

la sauvegarde, la valorisation, la revitalisation et principalement, la transcription du patrimoine linguistique kanak, ainsi que des expressions de tradition orale qui y 
sont associées, à travers notamment, la normalisation et la standardisation des 40 langues et dialectes kanak $^{17}$.

En outre, l'Académie dénombre officiellement 28 langues kanak et 11 dialectes ${ }^{18}$. Sachant que les formes linguistiques désignées par le terme dialecte sont dominées sur les plans numériques (nombre de locuteurs) et symboliques (reconnaissance sociale associée), elles donnent facilement lieu à des connotations péjoratives et à des dérives interprétatives. Dans les discours informels que nous pouvons observer en situation conversationnelle, des observateurs, dont des étudiants, en arrivent ainsi à disqualifier les langues kanak dans leur ensemble. Devant l'invisibilité susmentionnée de la diversité linguistique à Nouméa, il n'est pas rare en effet d'entendre des témoignages d'étonnements du type « 28 langues kanak ?! vraiment des langues, langues ou plus des dialectes?». Interrogés en début d'année universitaire sur leurs connaissances de langues autres que le français, certains étudiants répondent par exemple «non, juste des dialectes de chez moi ». À ces formes de mépris interlinguistique s'ajoute une attitude condescendante vis-à-vis des jeunes Kanaks dans leurs pratiques du français : « Tu parles bien français pour un Kanak qui a grandi dans les iles Loyauté » (photo ${ }^{\circ}$ 3). En arrière-plan, on refuse que le sujet n'ayant pas grandi dans un espace supposé générer un certain seuil de maitrise linguistique puisse en avoir les capacités, comme si la performance linguistique était conditionnée exclusivement par la fréquentation assidue de cet espace. En l'occurrence, ce serait en vivant à Nouméa, capitale de la Nouvelle-Calédonie, que d'aucuns apprendraient à mieux parler français. À contrario, ceux qui vivent dans les iles Loyauté ne sont pas supposés maitriser la langue française parce que l'on n'y parle pas la « vraie » langue ou l'on n'y parle tout simplement pas la langue. Le prisme du nativisme transparait alors. La tentative de cloisonnement géographique est manifeste dans ce type de remarque avec l'idée d'un cantonnement des individus dans des espaces ciblés. Dans un pays où l'on a pratiqué le régime de l'indigénat avec l'enclavement des Kanaks dans des réserves, cette micro-agression prend dès lors une dimension éminemment politique. En méprisant la qualité supposée de la ou des langues parlées dans l'espace d'origine du sujet, l'auteur de la microagression méprise in extenso le sujet lui-même. Parfois, le mépris est plus explicite : «C'est cool tu parles bien le français mais c'est ton accent qui fausse la note! (photo $\mathrm{n}^{\circ} 4$ ). Dans cette remarque, la forme syntaxique semble convenir à l'auteur de la microagression, mais c'est la prosodie qui est pointée. L'étudiante-destinataire pressent que l'auteur voit la langue comme un objet uniforme à différents niveaux: prosodique, phonologique, syntaxique, etc. Dans son descriptif, l'étudiante enregistre cet accent comme une marque identitaire qui la distingue des autres mais qui leur indique surtout des origines dont elle est fière. Supprimer cet accent consisterait à renier ses origines. 
Photo $n^{\circ} 3$.

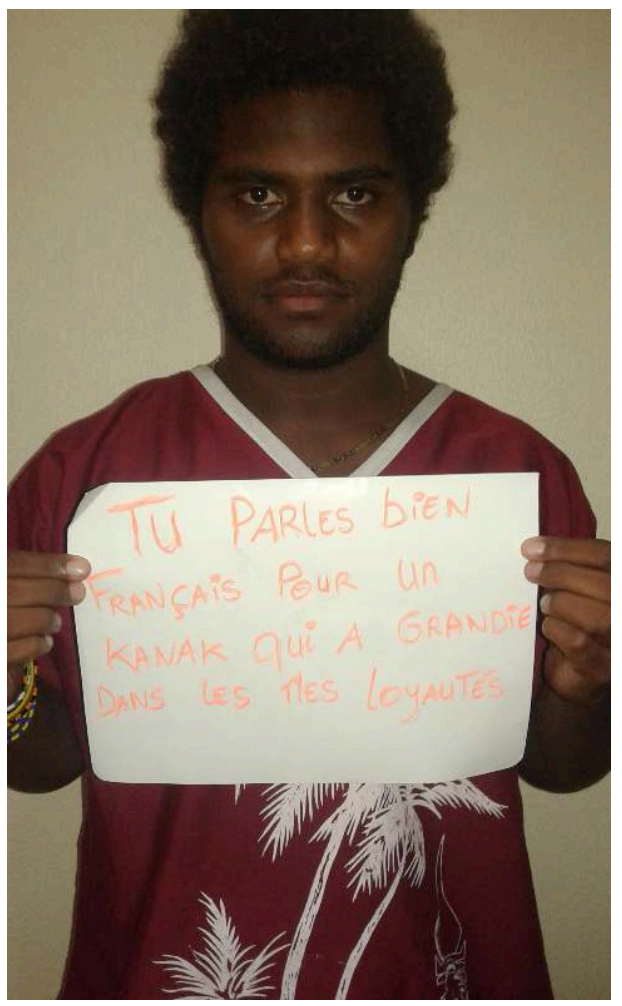

Photo ${ }^{\circ} 4$.

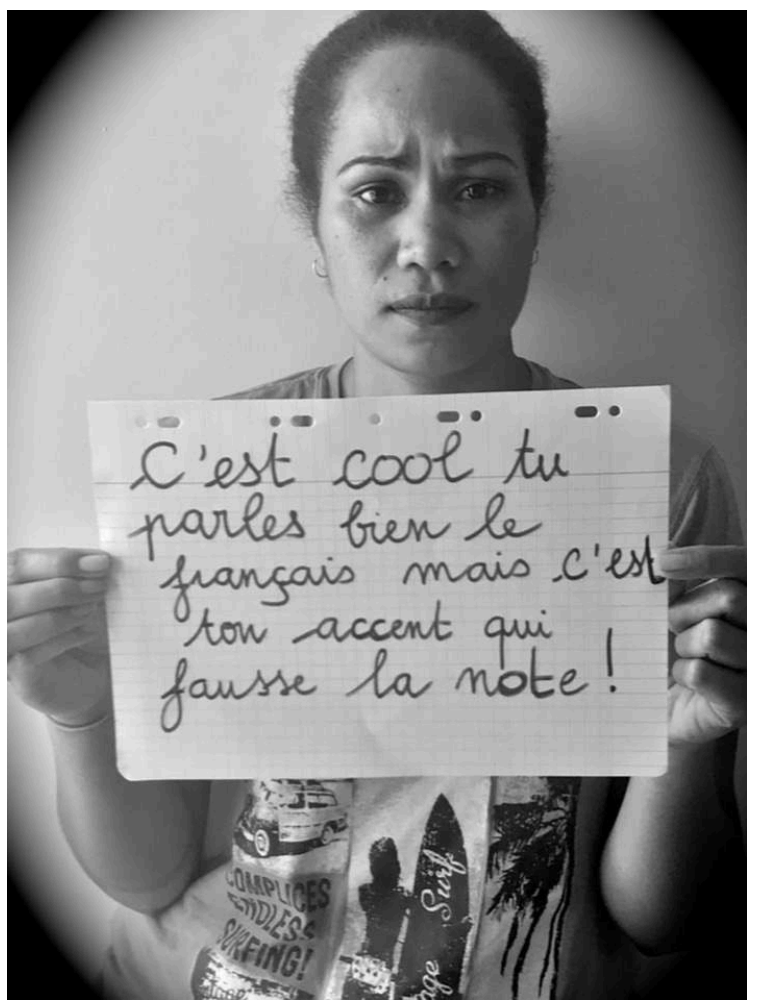

La deuxième forme de mépris s'observe à travers des micro-agressions intralinguistiques. Cette fois-ci, des phénomènes de rejet s'opèrent entre les membres 
d'une même communauté linguistique. Exemple récurrent en Nouvelle-Calédonie, une variété du drehu (langue de l'ile de Lifou, iles Loyauté) est perçue comme étant plus «pure» que les autres. Quel que soit le système linguistique mobilisé, les caractéristiques langagières estimées trop différentes de la norme essentialisée sont méprisées et conduisent à des jugements portés par les uns sur les pratiques des autres, dévaluées comme des performances au mieux incorrectes ("Ça ne te va pas de parler la langue », photo $n^{\circ} 5$ ), au pire intolérables ("Vaut mieux pas que tu parles en drehu », photo $n^{\circ} 6$ ). Dans la première remarque, le sentiment d'exclusion ne concerne pas seulement la communauté linguistique mais aussi la communauté elle-même qui est implicitement vue comme la masse silencieuse derrière ce "refus de reconnaissance » (Honneth, 1992, p.166). La deuxième remarque est symptomatique de ces microagressions qui dévaluent une production en langue et minent toute tentative d'expression orale.

Photo ${ }^{\circ} 5$.

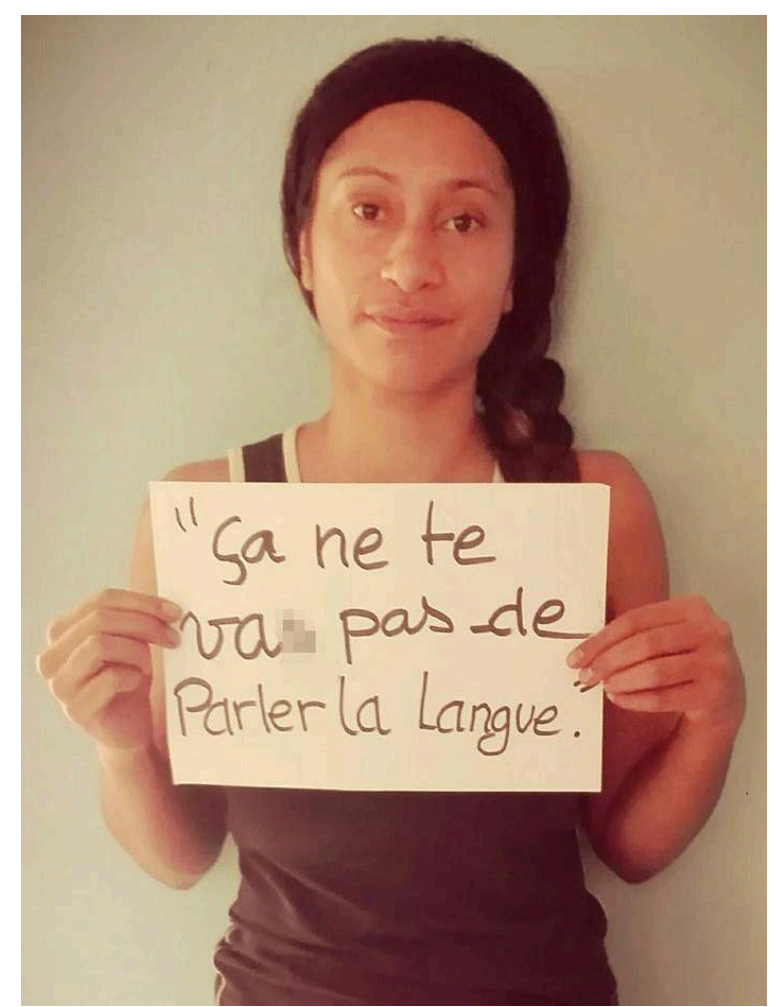


Photo ${ }^{\circ} 6$.

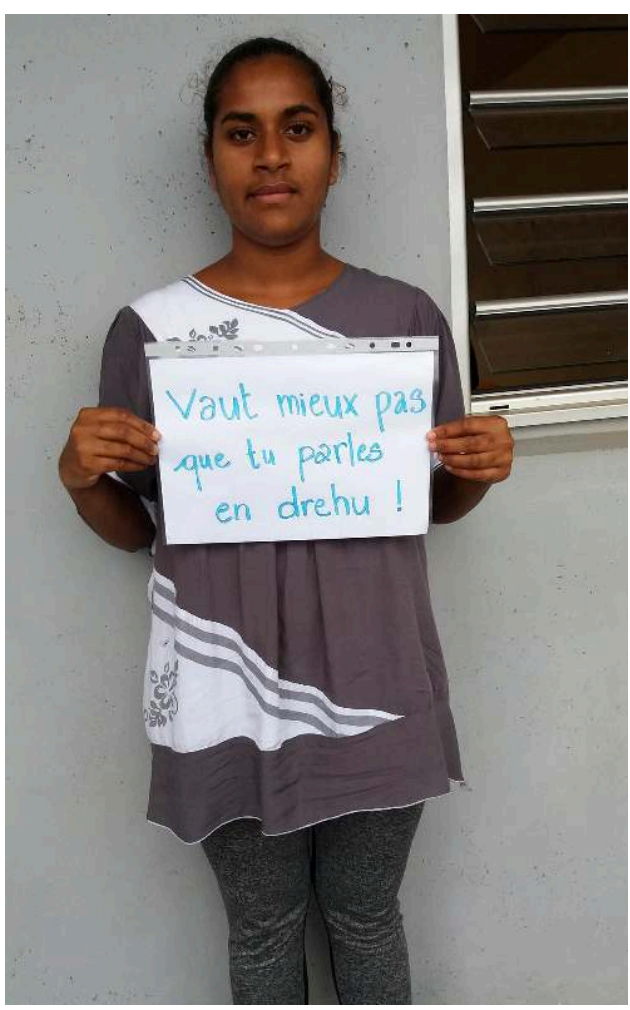

Enfin, le mépris linguistique peut résulter d'une concurrence entre les dynamiques plurilingues (pratiques, compétences, répertoires, identités) et celles associées au monolinguisme. Les pratiques plurilingues peuvent être assimilées à des transgressions. Assimilées à des "mélanges", elles symbolisent, aux yeux de leurs détracteurs, autant de l'ignorance intellectuelle qu'un déficit de légitimité : "Ça mélange le Maré et le Ouvéa et ça se dit mec de Lifou " (photo $n^{\circ} 7$ ). Le sentiment d'incompétence est construit par ce type de mépris linguistique alors même que les auto-dévalorisations des étudiants face à leurs propres aptitudes langagières sont indépendantes de leur niveau de maitrise linguistique. Qu'ils excellent en la matière ou pas, tous les étudiants ont fait part de micro-agressions débouchant sur une mauvaise estime de leur soi locuteur. Ne comprenant pas toujours les motivations à l'origine des micro-agressions dont ils sont les cibles, les étudiants en subissent parfois des effets de «honte»: «Cette micro-agression m'a handicapé: lorsque mes camarades me parlaient en drehu, je n'arrivais pas à leur répondre en langue et j'avais honte » (étudiante, Licence 2). C'est en mettant en récit leurs propres historicités langagières que les étudiants ont commencé à se distancier des injonctions intériorisées et des formes de mépris qu'elles ont autorisées. 


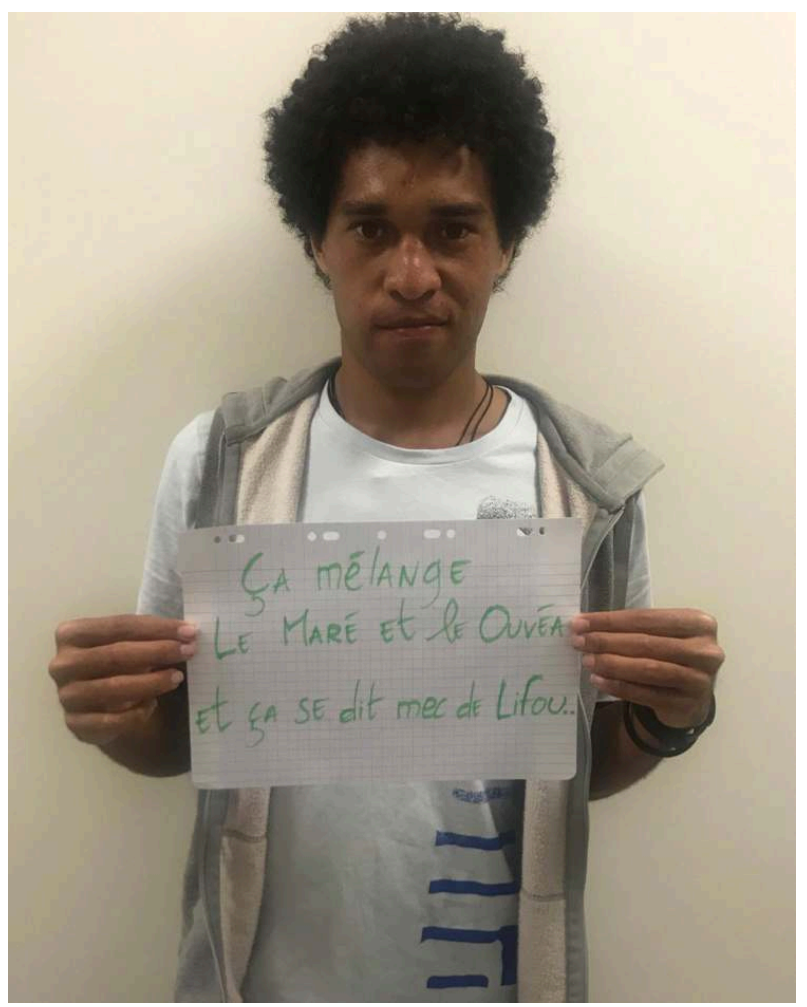

\section{Du mépris linguistique à l'émancipation linguistique}

Au regard de leurs récits, les étudiants commencent à mieux vivre leurs différences linguistiques dès lors qu'ils prennent conscience de la «normalité » du phénomène de la variation linguistique mais aussi de leurs propres compétences plurilingues et/ou pluriculturelles.

\subsection{L'interlangue, lieu de mépris et facteur d'émancipation}

27 Les variations interlinguistiques apportent leur lot de mépris: "Bordel, ka za la qene Lösi i ö! » «Bordel, c'est joli ton accent Lösi!» (photo $n^{\circ}$ 8). Dans son texte explicatif, l'étudiante fait part de sa perplexité face à l'ironie de cette remarque aux allures valorisantes mais qui se révèle moqueuse :

Lorsque je discute avec une personne, qui n'est pas de mon district, elle va m'observer, ensuite elle va rire sans que je la comprenne. Et donc, je vais m'apercevoir tout le long de notre discussion qu'elle était attirée par mon langage [...] je pense à une forme de micro-agression car c'est une forme de moquerie (remarque blessante) envers mon langage et ma façon de parler. (Étudiante, Licence 2)

En effet, la variante lösi peut être tournée en dérision par les locuteurs de Wetr et de Gaica, car elle est perçue comme étant davantage «noble », miny, c'est-à-dire parlée dans les cours des chefs ${ }^{19}$. La raison en est que la Bible a été traduite en cette variété, également acceptée comme étant la forme la plus ancienne. C'est d'ailleurs celle qui est enseignée aujourd'hui dans les écoles de Lifou et de la Province Sud. Le mythe nativiste apparait sous une forme différente. Le statut identitaire entre également en jeu. Du fait 
de sa proximité avec la langue noble, la jeune locutrice du lösi se retrouve au centre d'un amalgame. Sa façon de parler lui confère un statut qu'elle ne revendique pas. Elle ne comprend pas pourquoi elle est méprisée et finit par un certain mutisme ( Il m'arrive de ne plus parler ou de ne plus terminer mes phrases, tellement que je suis touchée »). En se rendant compte que la variation linguistique est non seulement une qualité inhérente à toutes les langues mais également une dynamique indispensable à leur survie, les étudiants assument plus facilement leurs pratiques langagières. Lorsqu'ils s'aperçoivent des compétences requises pour les productions plurilingues, nous observons des revendications de répertoires plurilingues là où, en début de programme, nous constations plutôt des stratégies d'invisibilisation.

Photo $\mathrm{n}^{\circ} 8$.

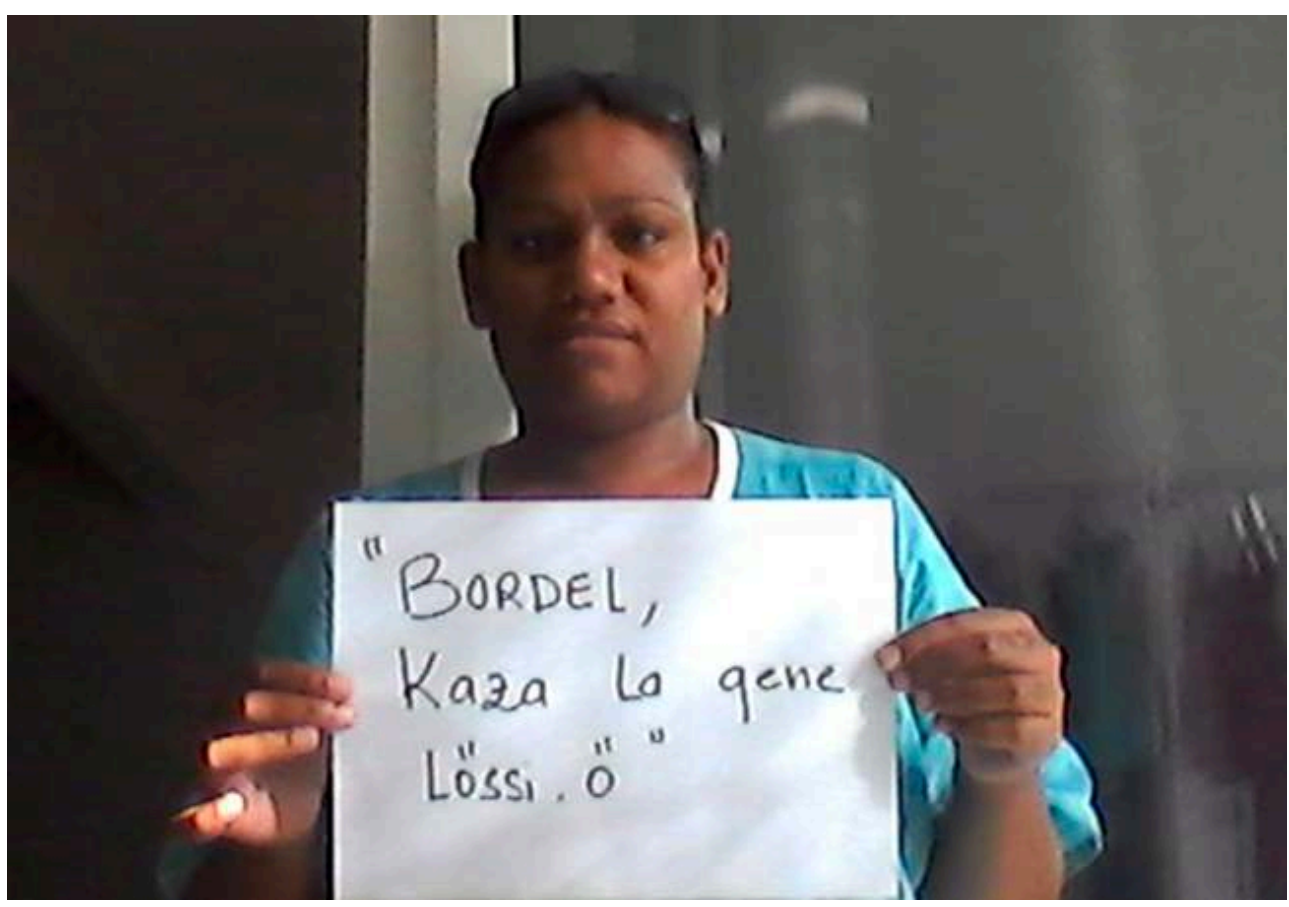

Une autre étudiante conclut son témoignage en racontant comment sa langue, autrefois interdite par l'école républicaine, est désormais assumée : «Aujourd'hui, ces mots font partie de mon vocabulaire usuel. Ils sont mon identité.» (Étudiante, Licence 3)

Autre concept aidant, celui de l'«interlangue» mis en lien avec les pratiques océaniennes. Basée sur une comparaison entre les systèmes phonologiques du français et ceux des langues kanak et polynésiennes, l'interlangue concerne aussi bien la prosodie que les phonèmes, la forme des mots, l'ordre syntaxique, etc. (Vernaudon, 2004, p.5). La linguistique descriptive démontre que toutes les langues n'ont pas recours au même «stock» de sons pour fabriquer des mots. Un substrat linguistique océanien peut ainsi être à l'origine de variations interlinguistiques. Dans les langues kanak, la variété intègre aussi une dimension régionale et statutaire. Une langue peutêtre différemment parlée par les tribus du bord de mer et par celles de la montagne. Elle sera marquée par des mots et par un accent différents. C'est le cas du drehu dont les pratiques sont distinctes selon que l'on est dans le sud de l'ile (district de Lösi) ou le nord (districts de Wetr et de Gaica). Dans la même perspective, l'étude des phénomènes de pluralité/diversité linguistique permet aux étudiants de renverser l'idée 
d'«interférences linguistiques» en celle de «compétences plurilingues et pluriculturelles» (Coste, Moore \& Zarate, 2009). Pour autant, cela ne suffit pas pour faire disparaitre les formes d'inhibitions provoquées par le mépris linguistique tant il détériore l'image de soi.

\subsection{L'art de la résilience linguistique}

31 Les étudiants décrivent les effets des micro-agressions linguistiques par l'hypercorrection, la peur de "faire des fautes", le stress communicationnel (gorge nouée, voix tremblante, etc.) et d'autres manifestations de l'insécurité linguistique. Une auto-dévaluation de leurs propres compétences s'observe également. L'estime de leur soi-locuteur étant ternie, c'est toute leur personne qui s'en trouve affectée. Nous avons donc œuvré à réhabiliter l'image plus largement sociale que les étudiants se faisaient d'eux-mêmes. Encouragés par de précédentes recherches qualitatives en sociolinguistique, nous avons fait le choix de passer par la photographie, en ce qu'elle "donne à voir un effet de sens " (Razafimandimbimanana, 2014, p. 57). La saisie photographique fige un espace-temps dans l'infini. Métaphoriquement, on peut y voir un figement du stigmate dans le passé comme pour mieux s'en décentrer et avancer.

Pour poursuivre dans ce processus de réhabilitation du sujet-locuteur et donner un peu plus d'ampleur à ce projet artistique, nous avons eu recours au street art. Nous voulions sensibiliser le public hors des espaces exclusivement textuels, académiques et scientifiques. Suite à l'idée originale de Paul Barri, alias PaBlöw, un artiste graffeur, Yann Skyronka, alias Sham Graff, a accompagné les étudiants dans un travail créatif. Les étudiants, à la fois porteurs de la diversité linguistique et acteurs de sa reconnaissance, ont ainsi personnalisé leurs autoportraits imprimés en grand format à l'aide de motifs librement apposés au feutre, guidés par leurs propres sources d'inspirations (photo $\mathrm{n}^{\circ}$ 9). Les affiches ont ensuite été collées sur différents murs de la ville (photo $\left.\mathrm{n}^{\circ} 10\right)$.

Outre la mise à distance que permet la recherche artistique, cette étape du projet symbolise également le moment où les étudiants se réapproprient (au sens propre comme au sens figuré) leur image sociale afin de pouvoir entamer la reconstruction d'une meilleure image de soi . Le temps d'un art éphémère, leurs réalités constituent le paysage discursif et visuel de la ville. Elles ont ouvert un dialogue aux sonorités régionales, aux contours plurilingues et aux graphies informelles. Avec les microagressions qu'elles donnent à voir, formes et fonds alimentent les réactions. Tous ont reçu des remarques (mono)normatives et correctrices à la suite d'une prise de parole. Ces remarques ont le pouvoir d'être imprévisibles. Paradoxalement, les plus destructrices sont souvent rampantes, aux apparences anodines. Il appartient au passant de s'en étonner ou pas. 
Photo $n^{\circ} 9$.

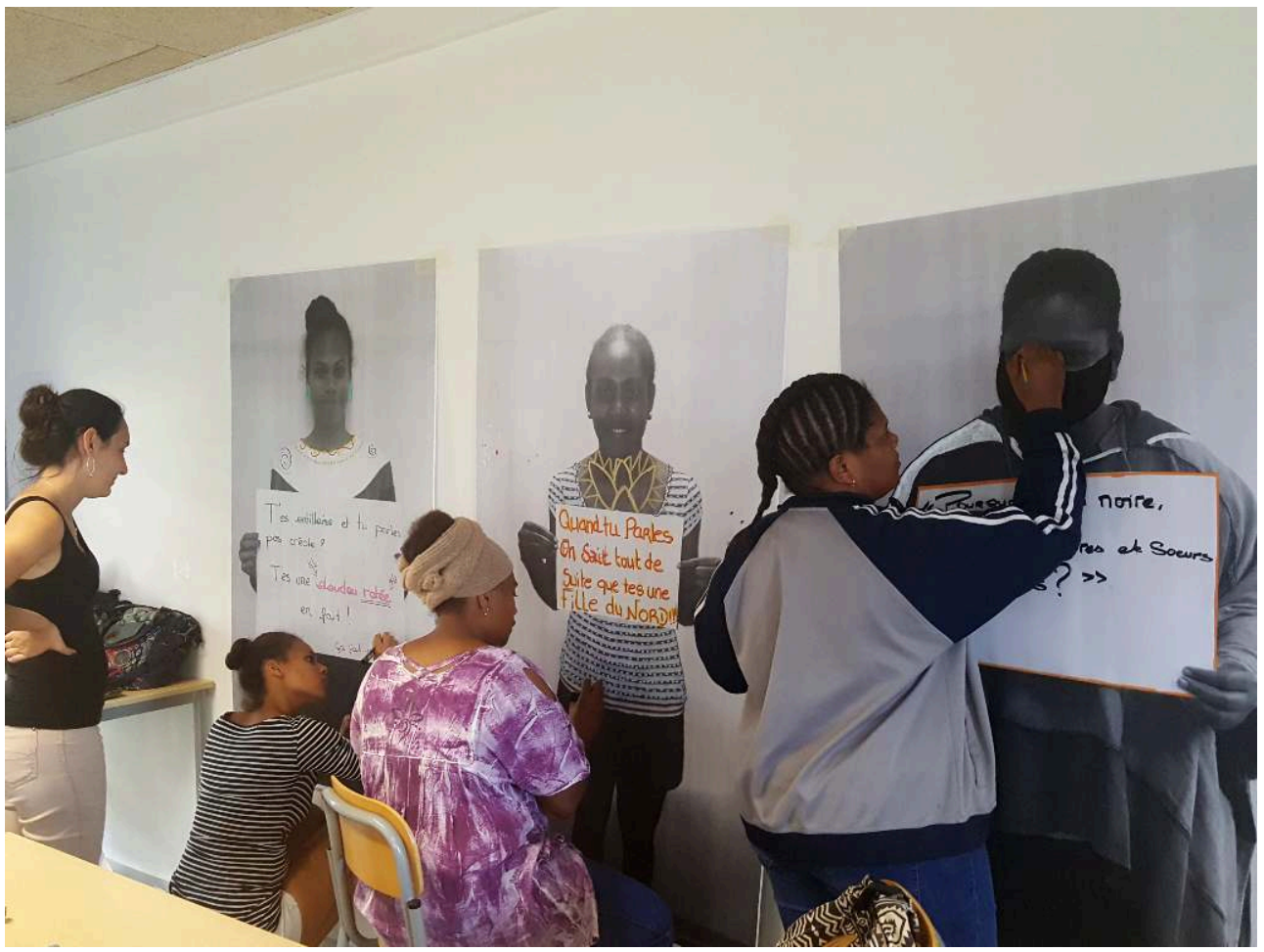

Photo $\mathrm{n}^{\circ} 10$

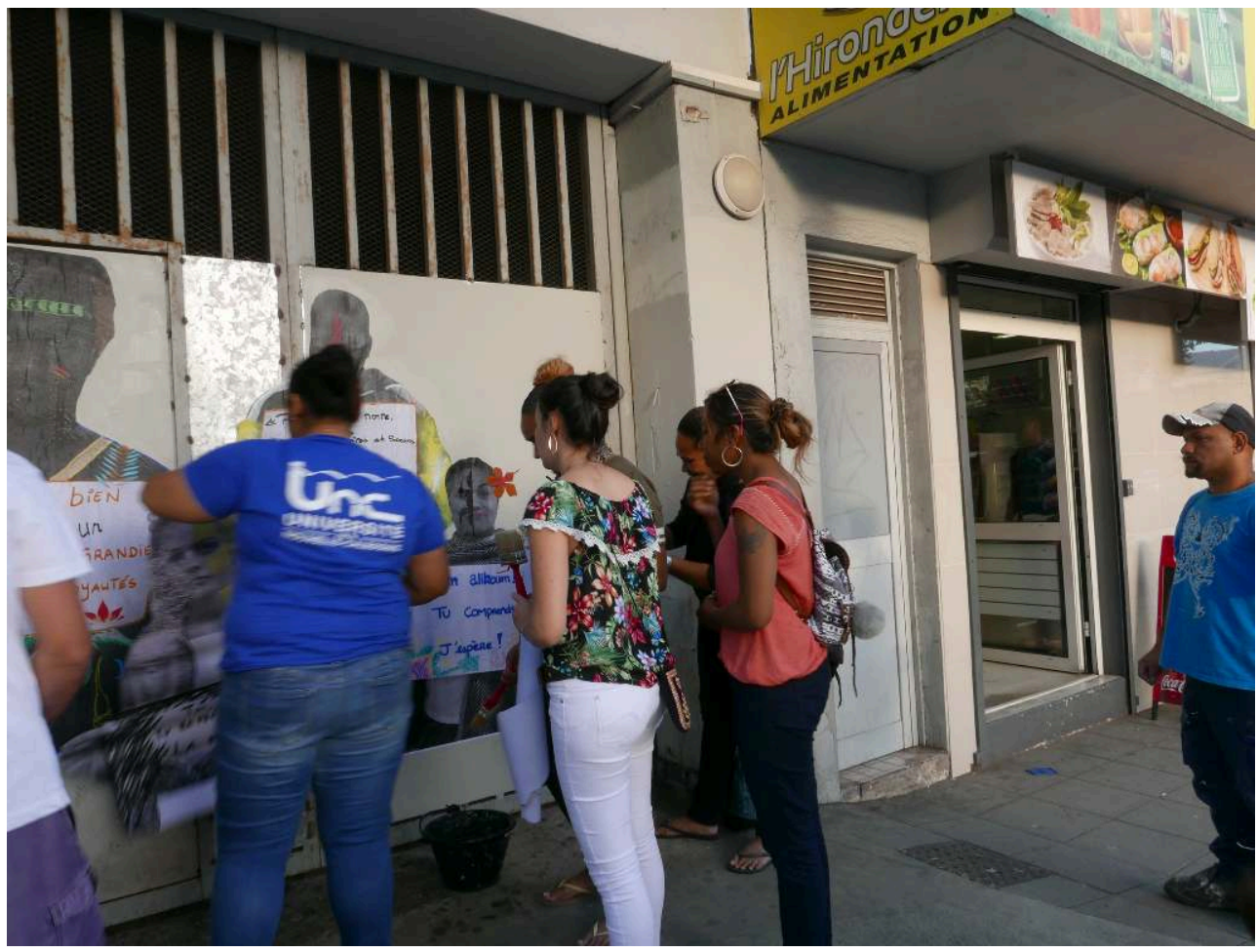




\section{Conclusion}

La Nouvelle-Calédonie revendique la pluralité culturelle de sa population et a mis à l'honneur sa richesse linguistique dans les accords politiques ${ }^{20}$ qui l'ont conduite sur le chemin de l'autogestion. Cela a permis d'atténuer certaines représentations jusque-là dégradantes sur les langues kanak et le bi/plurilinguisme. La diversité linguistique, désormais actée sur le plan légal, reste contestée lorsqu'elle prend la forme de pratiques bi/plurilingues. Le mépris linguistique, s'il est marqué dans le discours, s'opère moins de manière frontale que de façon indirecte selon les témoignages des jeunes participants au projet AK-100. Le concept de "micro-agressions linguistiques » permet de rendre compte de remarques vécues comme étant insidieusement dévalorisantes. Le caractère insidieux tient soit à un discours d'apparence flatteuse, soit au processus latent mais néanmoins agissant des discours correcteurs, mononormatifs, ethnicisants, disqualifiants, etc. Cette forme de mépris linguistique débouche sur un sentiment d'insécurité qui transcende le fait linguistique en ravivant ses autres expériences de rejet social. Dans leurs différents écrits réflexifs, les étudiants parlent souvent d'évènements datant de leur scolarité, remontant parfois jusqu'au primaire, et dont les effets négatifs sont encore présents à l'université. Ils sont effectivement destructeurs en ce qu'ils minent le sujet-locuteur et l'inhibent dans ses prises de parole ainsi que dans ses choix de transmission linguistique :

Je vis chaque jour avec la crainte de mal m'exprimer, qu'on se moque de moi

et surtout de ne pas pouvoir transmettre à mon fils cette richesse ${ }^{21}$.

(Étudiante, Licence 2)

À travers le projet AK-100, les étudiants ont notamment pris conscience de la «normalité » des variations inter/intralinguistiques et des compétences requises pour les pratiques bi/plurilingues. Couplé à l'action street art, le travail autour des microagressions linguistiques a participé à la reconstruction de meilleures images de soi. L'un des signes de l'émancipation des jeunes par rapport au mépris linguistique est la manière dont ils se sont saisis des espaces publics ou médiatiques pour s'afficher, s'opposer, en un mot, s'assumer. Une des participantes s'est ainsi portée volontaire pour témoigner des «micro-agressions linguistiques» dans une émission télévisée ${ }^{22}$. À cette occasion, les chroniqueurs ont également affiché, à leur tour, les remarques stigmatisantes dont ils ont été la cible (photo $\mathrm{n}^{\circ} 11$ ). 
Photo $\mathrm{n}^{0} 11$.

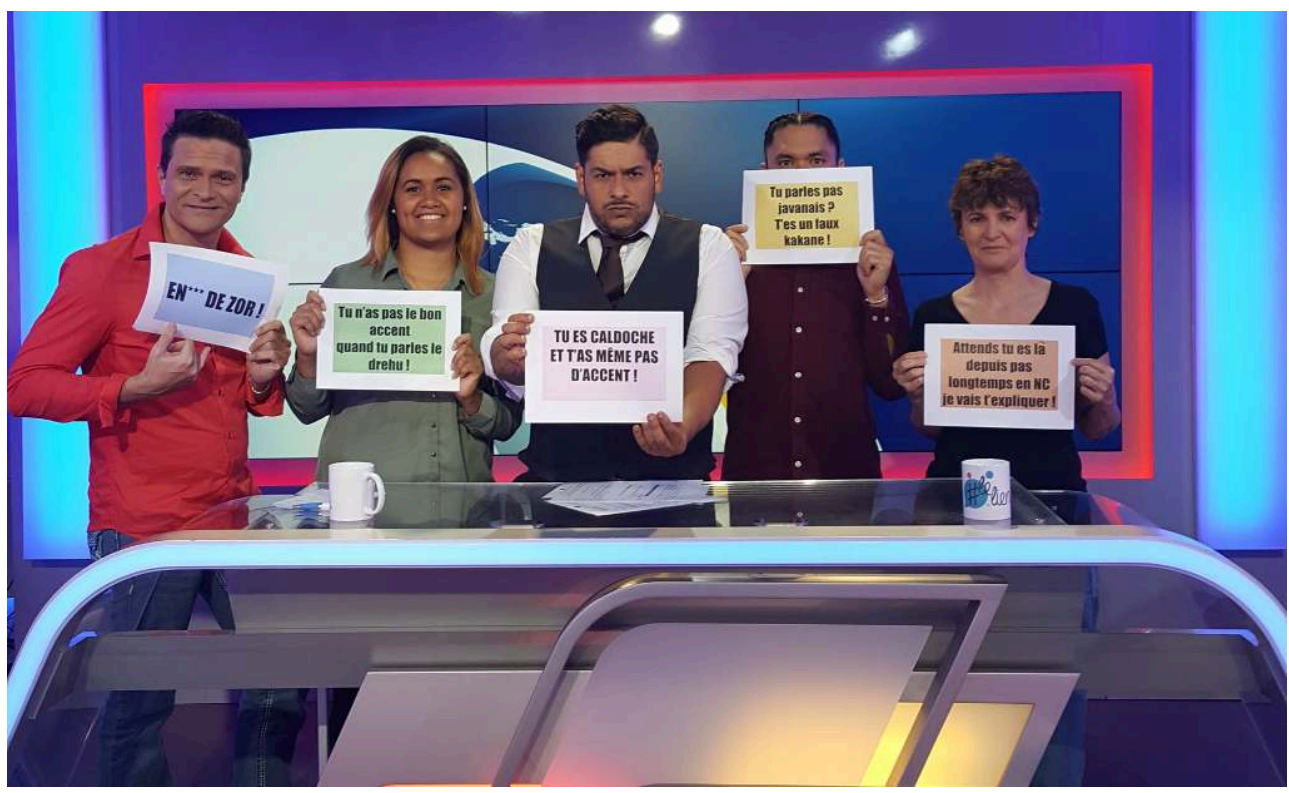

D'autres se sont lancés dans des concours d'éloquence comme réaction face aux critiques reçues. Plusieurs parmi ceux-ci ont d'ailleurs choisi de traiter de la discrimination linguistique et de ses effets bloquants dans leur performance. Ce fut le cas de la lauréate du concours 2018, également remarquée pour sa prestation sur le droit de mobiliser deux langues lors du Grand oral (France 2), début $2019^{23}$. Le mépris est toujours une réalité pour les étudiants, mais ils savent désormais l'expliquer, ce qui facilite la déconstruction de ses effets. La résilience apparait aussi dans les projets qu'ils énoncent. Il est remarquable que leurs motivations passent d'une critique subie personnellement (photo $\mathrm{n}^{\circ} 11$ ) à un élan bienveillant et collectif :

J'ai choisi cette phrase, car je ne suis pas locutrice de mes deux langues d'origine.

Ces deux langues sont le pije et le vamale.

Le pije et le vamale sont des dialectes parlés dans les régions de Hienghène et Touho, le pije est en voie d'extinction, seuls les plus âgés parlent cette langue, quelques générations après, les gens les comprennent mais ne les parlent pas, d'ici une dizaine d'années, cette langue risque de disparaitre.

Pour le vamale ma génération ne parle pas mais la comprend, seules les générations avant nous l'emploient de manière très active. Quelques générations après, nous l'employons mais elle n'est pas aussi bien maitrisée que par les plus anciens.

À travers ce message, j'aimerais sensibiliser mes petits frères et petites sœurs ${ }^{24}$ qui viendront derrière moi, qu'ils puissent les préserver et les faire apprendre à leurs futurs enfants pour faire perdurer nos langues mais aussi pour qu'ils prennent conscience que ces langues font partie de notre identité, notre patrimoine kanak et qu'il est important de les préserver. Ce message s'adresse aussi à toutes les personnes qui se reconnaitront dans la même situation linguistique.

Pour ma part, c'est un message de tristesse, car je ne parle aucune de mes deux langues. Aujourd'hui j'essaye par mes propres moyens de les comprendre et de les parler car je pense qu'il n'est jamais trop tard pour les apprendre. (Étudiante, Licence 3) 


\section{BIBLIOGRAPHIE}

BARNÈCHE, Sophie. (2005). Gens de Nouméa, gens des îles, gens d'ailleurs. Langues et identités en NouvelleCalédonie. Paris : L'Harmattan.

BLANCHET, Philippe. (2016). Discriminations : combattre la glottophobie. Paris : Éditions Textuel. BochmanN, Klaus. (2001). Notre langue, votre patois, leur baragouin : stéréotypes et représentations des langues. Hermès, 30, 91-102.

Сномsку, Noam. (1971). Aspects de la théorie syntaxique (J.-C. Milner, trad.). Paris : Le Seuil. (Ouvrage original publié en 1965 sous le titre Aspects of the Theory of Syntax. Cambridge : MIT Press.)

Colombel, Claire \& Fillol, Véronique. (2012). Enjeux des langues d'enseignement dans la construction d'une identité scolaire des jeunes océaniens francophones. Dans F. Demougin \& J. Sauvage (dir.), La construction identitaire à l'école. Perspectives linguistiques et plurielles (p. 113-120). Paris : L'Harmattan.

COSTE, Daniel, MOORE, Danièle \& ZARATE, Geneviève. (2009 [1997]). Compétence plurilingue et pluriculturelle. Vers un Cadre européen commun de référence pour l'enseignement et l'apprentissage des langues vivantes. Strasbourg : Division des Politiques linguistiques.

DoтTE, Anne-Laure. (2019, juin). Moi je parle pas la vraie langue! Quelle(s) valeur(s) pour quelle(s) variation(s) linguistique(s) ? Communication présentée au $4^{\mathrm{e}}$ Congrès du Réseau francophone de sociolinguistique « Langues de valeur et valeurs de langues », Ottawa, Canada.

FiLlol, Véronique \& VeRnAUdon, Jacques. (2011). Toutes les langues à l'école. Enseignement des langues kanak et éveil aux langues de la région Asie-Pacifique à l'école calédonienne. Dans F. Laroussi \& F. Liénard (dir.), Plurilinguisme, politique linguistique et éducation : quels éclairages pour Mayotte? (p. 203-213). Mont-Saint-Aignant : Presses universitaires de Rouen et du Havre.

Frain, Sylvia, RAZAFIMANDIMBIMANANA, Elatiana \& WACALIE, Fabrice. (2019). Speaking Back: Challening Linguistic Microaggressions in New Caledonia through Street Art. Disponible en ligne sur $<$ www.pasifikarising.org/speaking-back-challenging-linguistic-microaggressions-in-newcaledonia-through-street-art/> (consulté le 21 septembre 2019).

HoNNETH, Axel. (1992). La lutte pour la reconnaissance (P. Rusch, trad.). Paris : CERF. (Ouvrage original publié en 1992 sous le titre Kampf um Anerkennung. Berlin : Suhrkamp.)

HonNETH, Axel. (2006). La société du mépris : vers une nouvelle théorie. Paris : La Découverte.

MUNI TOKE, Valelia. (2013). Le locuteur natif et son idéalisation : un demi-siècle de critiques. Histoire Épistémologie Langage, 35(2), 5-15.

PHILLIPSON, Robert. (1992). Linguistic Imperialism. Oxford : Oxford University Press.

Pierce, Chester, CAReW, Jean, Pierce-Gonzalez, Diane \& Willis, Deborah. (1978). An Experiment in Racism: TV Commercials. Dans C. Pierce (dir.), Television and Education (p. 62-88). Beverly Hills : Sage.

RAZAFIMANDIMBIMANANA, Elatiana. (2014). Quelque part entre des inaccessibles : une façon de penser la photographie et le sens en sociolinguistique. Glottopol, 23, 47-76. 
RAZAFIMANDIMBIMANANA, Elatiana. (2017, octobre). Paradis pour la diversité linguistique : la NouvelleCalédonie ? Communication présentée à l'Université de Montréal, Canada.

RAZAFIMANDIMBIMANANA, Elatiana. (à paraitre). La diversité linguistique à l'école : représentations d'élèves en Nouvelle-Calédonie. Dans R. Ailincai \& S. Ferrière (dir.), École et famille, Langues et cultures en Océanie : regards croisés. Nouméa : PUNC.

RAZAFIMANDIMBIMANANA, Elatiana \& WACALIE, Fabrice. (2019). Les micro-agressions linguistiques. Hermès, 83, 156-157.

RAZAFIMANDIMBIMANANA, Elatiana \& FAVARD, Nicolas. (2018). Les élèves aux besoins éducatifs particuliers se mettent en représentation : «On parle plusieurs langues pour progresser ». Dans Actes du séminaire CREFAP/OIF. Enseignement et formation du/en français en contexte plurilingue (p. 311-335). Hanoï : Éditions de l'Université nationale du Vietnam.

SALAÜN, Marie. (2005). L'école indigène, Nouvelle-Calédonie, 1885-1945. Rennes : Presses universitaires de Rennes.

SKUTNABB-KANGAS, Tove. (1986). Multilingualism and the Education of Minority Children. Dans R. Phillipson \& T. Skutnabb-Kangas (dir.), Linguicism Rules in Education (p. 42-72). Roskilde, Danemark : Roskilde University Centre Institute.

SuE, Derald Wing, CAPOdiluPo, Christina M., ToRino, Gina C. \& BucCeri, Jennifer M. (2007). Racial Microaggression in Everyday Life. Implications for Clinical Practice. American Psychologist, 62(4), 271-286.

VERNAUDON, Jacques. (2004). Grammaire comparée des langues océaniennes et de la langue française. Disponible en ligne sur <http://www.ac-noumea.nc/IMG/pdf/gram_comp2.pdf> (consulté le 21 septembre 2019).

VERNAUDON, Jacques. (2013). L'enseignement des langues kanak en Nouvelle-Calédonie. Hermès, 65(1), 112-118.

WACALIE, Fabrice. (2006). Étude des marqueurs aspectuels a, ka et kola/kolo en drehu (Lifou, Iles Loyautés) (Mémoire de master non publié). Institut national des langues et civilisations orientales, Paris.

\section{NOTES}

1. Nous employons les termes langue et linguistique au sens large en désignant indifféremment les systèmes et les variétés de langues.

2. La Nouvelle-Calédonie et la Polynésie française possèdent le statut de collectivité sui generis. Ce terme latin signifiant «de son propre genre » est attribué aux territoires qui jouissent d'une situation juridique particulière, de dérogations à certains grands principes et de transfert de compétences.

3. Sans distinction normative entre ce qui relèverait de « langues » versus des « dialectes ».

4. Il s'agit de l'année où la loi Deixonne, votée en 1951 en France pour l'inclusion des langues régionales, a été étendue aux langues kanak de la Nouvelle-Calédonie.

5. La présente analyse survient après le référendum sur l'indépendance de novembre 2018, évènement indissociable des questions identitaires que représentent les langues. Néanmoins, nous pensons que le référendum n'a pas eu une influence significative sur les phénomènes de réappropriation linguistique qui s'inscrit plutôt dans un processus identificatoire longitudinal de 
prise de conscience (individuelle et collective) quant à la nécessité de s'émanciper du modèle monolingue ou du «tout-français ».

6. Discours récurrent relevé dans des portfolios réflexifs réalisés par les étudiants dans le cadre de projets pédagogiques.

7. Capitale de la Nouvelle-Calédonie.

8. Cette question vaut pour le cours de Psycholinguistique et le parcours Didactique du plurilinguisme.

9. «Parler la langue » ou " parler en langue » renvoie à une expression courante utilisée pour désigner une ou les langue(s) kanak.

10. Notre traduction.

11. Notre traduction.

12. C'est-à-dire complimenter, faire rire, dire des évidences sociales pour détendre l'ambiance, partager une observation qui parait évidente, etc.

13. Gras de l'étudiante.

14. Quelques étudiants ont proposé deux portraits illustrant deux remarques distinctes.

15. Échanges informels hors consignes de travail.

16. Discours type transcrit à partir de nos observations-participantes in situ. Voir la note 5.

17. <www.alk.nc/academie-des-langues-kanak/qui-sommes-nous>.

18. Voir la note 5 .

19. Il existe en drehu deux registres de langue : l'une dite «standard » parlée par l'ensemble de la population ; l'autre, dite «noble ", le miny en langue drehu, utilisé pour s'adresser au chef et à sa cour (Wacalie, 2006). C'est dans cette variante que les missionnaires ont fait le choix de traduire la Bible.

20. Accords de Matignon (1988) ; accords de Nouméa (1998).

21. Gras de l'étudiante.

22. Émission \#lelien sur Nouvelle-Calédonie La Première, émission du 5 octobre 2018. <https:// la1ere.francetvinfo.fr/nouvellecaledonie/emissions/lien>.

23. <https://https://youtu.be/Pd9Fct00UZU>.

24. À entendre au sens inclusif des plus jeunes générations sans qu'il n'y ait nécessairement le lien biologique de la fratrie d'une famille nucléaire.

\section{RÉSUMÉS}

Dans les langues kanak, la même formule exprime les verbes parler et être. L'expression qene drehu signifie "parler drehu» et "être drehu», illustrant la dimension éminemment sociale de la langue. Il en est question dans cet article dédié au mépris vécu sous la forme de "microagressions linguistiques » par des étudiants de la Nouvelle-Calédonie. Ces micro-agressions sont des remarques insidieuses reçues comme un refus de reconnaissance quant à des compétences linguistiques, mais aussi quant au statut de locuteur légitime. Elles construisent et reproduisent implicitement des inégalités sociales en dévalorisant l'altérité linguistique. Les sentiments d'appartenance sociale et de bien-être s'en trouvent affectés et cèdent la place à l'autodévalorisation. Nous nous pencherons sur la façon dont elles se présentent et sur leurs principaux effets. Nous verrons aussi comme un travail de street art a pu enclencher un processus d'émancipation. 
In Kanak languages, the same expression is used for the verbs "to speak" and "to be". In Drehu, qene drehu means both "to speak Drehu" and "to be Drehu", which shows how languages are embedded in the way of being, the very topic of the present article: implicit forms of contempt as made witnessed by students in New Caledonia. Our research deals with "linguistic microaggressions", namely insidious comments perceived as the non-recognition of linguistic skills and legitimacy. Such comments construct and reproduce social inequality by devaluing linguistic alterity. Social belongingness and well-being thereby yield to low self-esteem. We will study their discursive forms and social effects. We will also see how street art triggered a process of resilience.

INDEX

Mots-clés : mépris, langues kanak, français, insécurité linguistique, micro-agressions linguistiques

Keywords : contempt, Kanak languages, French, linguistic insecurity, linguistic microaggressions

\section{AUTEURS}

\section{ELATIANA RAZAFI}

Université de la Nouvelle-Calédonie

\section{FABRICE WACALIE}

Université de la Nouvelle-Calédonie 